\title{
Synthesis and evaluation of new nifurtimox-adamantane adducts with trypanocidal activity
}

\author{
Angeliki-Sofia Foscolos, ${ }^{[a]}$ Ioannis Papanastasiou, ${ }^{[a] *}$ Andrew Tsotinis, ${ }^{[a]}$ Martin C. Taylor, ${ }^{[b]}$ and John \\ M. Kelly ${ }^{[b]}$
}

In memory of Professor George B. Foscolos, Department of Pharmacy, National and Kapodistrian University of Athens

[a] Ms. A.-S. Foscolos, 0000-0001-8123-5060; Asst. Prof. I. Papanastasiou, 0000-0001-6974-2561; Prof. A. Tsotinis, 0000-0002-4334-4810 School of Health Sciences, Department of Pharmacy, Division of Pharmaceutical Chemistry National and Kapodistrian University of Athens

Panepistimioupoli-Zografou, 15784 Athens, GREECE

E-mail: papanastasiou@pharm.uoa.gr

[b] Assoc. Prof. M.C. Taylor, 0000-0003-4147-0693; Prof. J.M. Kelly, 0000-0003-4305-5258

Department of Pathogen Molecular Biology, London School of Hygiene and Tropical Medicine Keppel Street, London WC1 E7HT, UK

Supporting information for this article is given via a link at the end of the document.

Abstract: The synthesis and pharmacological evaluation of the $\mathrm{C}-1$ substituted adamantane hydrazones 1a-d, their C-2 substituted isomers $\mathbf{2 a}-\mathbf{d}$ and the $\mathrm{C}-1$ substituted adamantane furanoic carboxamides $3 \mathbf{a}-\mathbf{c}$ is described. The new adamantane derivatives exhibited an interesting pharmacological profile, in terms of trypanocidal activity and selectivity. Of the compounds tested, the phenylacetoxy hydrazone $1 \mathrm{~b}$ showed the most promising profile against African trypanosomes $\left(\mathrm{EC}_{50}=11 \pm 0.9 \mathrm{nM} ; \mathrm{Sl}_{\mathrm{Tb}}=770\right)$. substituted adamantane ring. The adamantane core of the nove adducts accommodates aromatic substitutions at the C-1 and C2 positions. In addition, the 5-nitro-2-furanyl pharmacophore group is attached to the phenyladamantane by a hydrazone bond with a linker, consisting of one or two methylene groups and an oxygen atom. This bond is altered to carboxamide in compounds 3a-c with compound $3 \mathrm{c}$ also bearing a benzyl group on the amide nitrogen, to mimic the benznidazole skeletal arrangement (Figure

Vector-borne kinetoplastid diseases, such as Chagas disease (CD) ${ }^{[1]}$ leishmaniasis and human African trypanosomiasis (HAT) [2] threaten almost a billion people worldwide. ${ }^{[3]}$ The available drugs against these neglected tropical diseases (NTDs) are characterized by toxicity, limited efficacy and increasing resistance. ${ }^{[4]}$ For example, nifurtimox and benznidazole which are used against $C D$, can cause severe side-effects and treatment is often unsuccessful. ${ }^{[5]}$ This has led the World Health Organization (WHO) to coordinate public sector and private partnerships as part of a global effort to develop new and safer dugs. ${ }^{[3]}$ Fexinidazole, which was recommended by the European Medicines Agency in November 2018, is a successful example of the collaboration between the Drugs for Neglected Diseases initiative (DNDi) and pharmaceutical chemistry sector. ${ }^{[6]}$

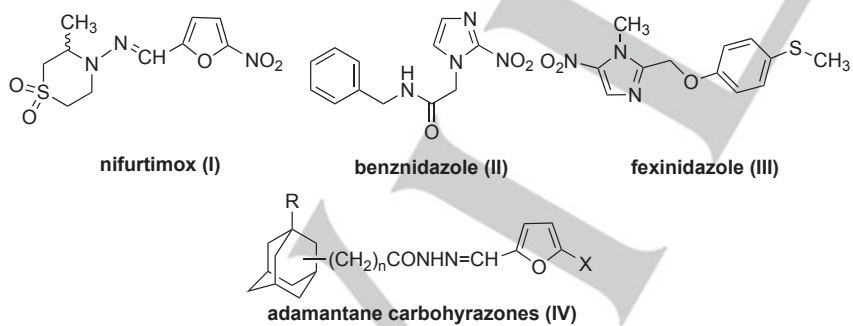

Figure 1. Nifurtimox, benznidazole, fexinidazole and adamantane carbohydrazone derivatives.

We have been interested in adamantane chemistry ${ }^{[7-21]}$ and have prepared a large number of analogues in an attempt to exploit adamantane's role in bioactivity. We prepared a series of adamantane carbohydrazones, ${ }^{[22]}$ and showed that these derivatives are very potent trypanocidals. Following this work, we now describe the preparation of a series of phenylhydrazone analogues, $\mathbf{1 a - d}$ and $\mathbf{2 a - d}$, which in general have very promising antitrypanosomal activity. The new derivatives share common structural features with nifurtimox and also contain a phenyl

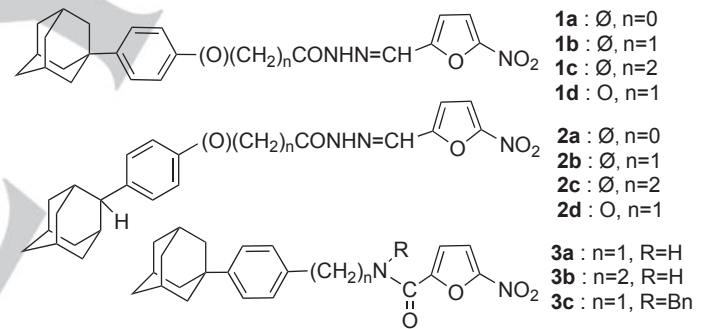

The 4-(adamant-1-yl)phenyl)hydrazides, 1a-d, were prepared by previously described methods, [22] using as starting materials ethyl 4-(adamant-1-yl)benzoate (2), [18] ethyl 4-(adamant-1yl)phenyl acetate (4), [18] ethyl 4-(adamant-1-yl)phenylpropionate (6) ${ }^{[18]}$ and ethyl 4-(adamant-1-yl)phenoxyacetate (8) ${ }^{[23]}$ as shown in Scheme 1.

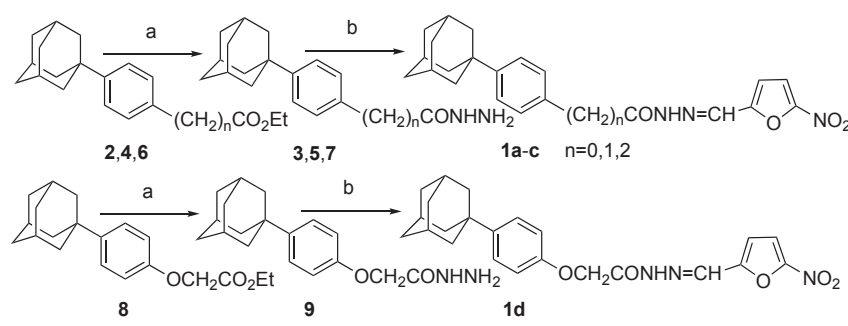

Scheme 1. Reagents and conditions: (a) hydrazine hydrate, $\mathrm{EtOH}, 3: 160{ }^{\circ} \mathrm{C}$ autoclave, $6 \mathrm{~d}, 81 \%, 5: 130{ }^{\circ} \mathrm{C}$, autoclave, $5 \mathrm{~d}, 85 \% ; 7$ : reflux, $2 \mathrm{~d}, 80 \%, 9$ reflux, 1d, $98 \%$;(b) 5-nitro-2-furaldehyde, EtOH, r.t., 12 h, 55-80 \%.

The preparation of analogues $2 \mathrm{a}-\mathrm{c}$ was realised by the reaction sequence illustrated in Scheme 2.
Figure 2. New nifurtimox-adamantane adducts with trypanocidal activity. 


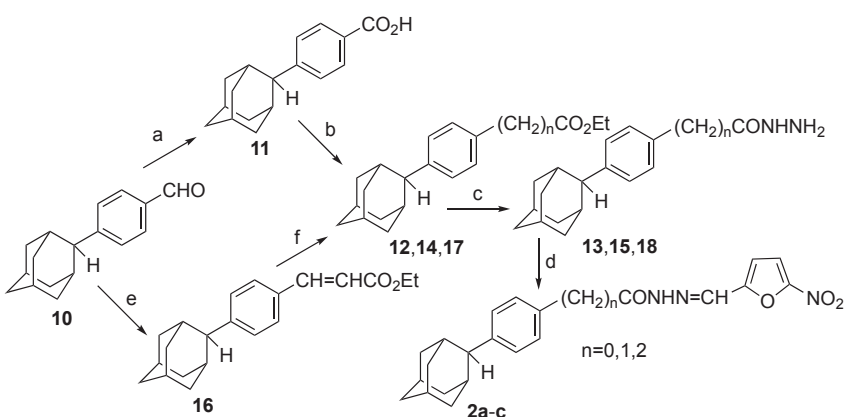

Scheme 2. Reagents and conditions: (a) Jones reagent $8 \mathrm{~N}$ in acetone, $3 \mathrm{~h}, 40 \%$; (b) i. $\mathrm{SOCl}_{2}$, gentle reflux, 60 min ii. EtOH (abs.), gentle reflux, $90 \mathrm{~min}, 79 \%$; (c) hydrazine hydrate, $\mathrm{EtOH}, 13: 160{ }^{\circ} \mathrm{C}$, autoclave, $6 \mathrm{~d}, 55 \%, 15: 160{ }^{\circ} \mathrm{C}$, autoclave, 6 d, $72 \%, 18$ : reflux, 1 d, $79 \%$; d) 5-nitro-2-furaldehyde, EtOH, r.t.,12 h, 39-82 \%; (e) i. triethyl phosphonoacetate and $\mathrm{NaH}$, anh. $\mathrm{THF}, 0^{\circ} \mathrm{C}, \mathrm{Ar}, 30 \mathrm{~min}$ and then at r.t., $60 \mathrm{~min}$ ii. saturated ammonium chloride solution, $0{ }^{\circ} \mathrm{C}, 66 \%$; (f): $\mathrm{H}_{2} / \mathrm{PtO}_{2}$, EtOH, 40 psi, r.t., $3 \mathrm{~h}, 91 \%$.

4-(2-Adamantyl)benzaldehyde (10) ${ }^{[17]}$ was oxidised by the Jones reagent to the corresponding benzoic acid 11, which was esterified to the respective benzoate 12. Under Emmons-Horner reaction conditions benzaldehyde 10 afforded the trans-ethyl cinnamate 16, which was then hydrogenated to the corresponding saturated propionate 17. Ethyl 4-(adamant-2-yl)benzoate (12), ethyl 4(adamant-2-yl)phenylacetate (14) ${ }^{[17]}$ and ethyl 4-(adamant-2yl)phenylpropionate (17) gave the desired hydrazones 2a-c via standard procedures.

The synthesis of the 2-(4-(adamant-2-yl)phenoxy)- $N$ '-((5nitrofuran-2-yl)methylene)acetohydrazide $\quad(\mathbf{2 d})$ is shown in Scheme 3.

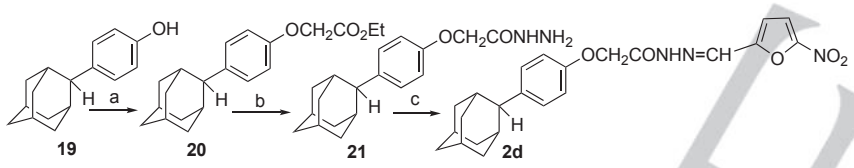

Scheme 3. Reagents and conditions: (a) $\mathrm{ClCH}_{2} \mathrm{CO}_{2} \mathrm{Et}, \mathrm{K}_{2} \mathrm{CO}_{3}$, dry DMF, $83 \%$; (b) hydrazine hydrate, EtOH, reflux, 1 d, 98 \%; (c) 5-nitro-2-furaldehyde, EtOH, r.t., 12 h, $92 \%$

The 4-(2-adamantyl)phenol (19), ${ }^{[24,25]}$ was ethoxycarbonylomethylated in the presence of potassium carbonate in dry DMF to afford the respective phenoxyacetate 20 , which led to the corresponding hydrazone $\mathbf{2} \mathbf{d}$ following the above methods. Due to the inefficiency of the literature methods for the preparations of 4-(2-adamantyl)phenol (19), we developed the alternative synthetic routes to 19 , shown in Scheme 4.

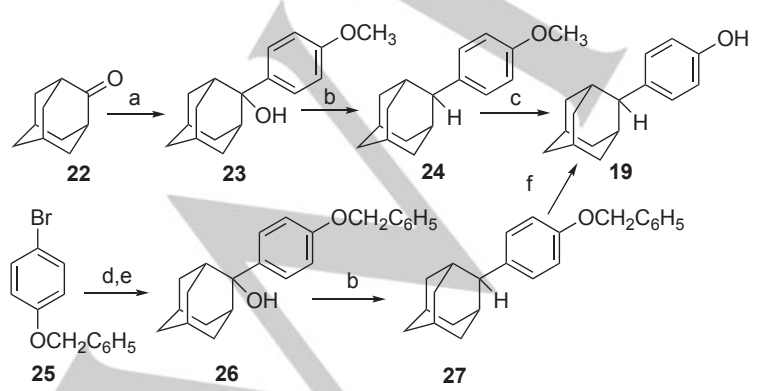

Scheme 4. Reagents and conditions: (a) i. 4-methoxyphenylmagnesium bromide, $\mathrm{Et}_{2} \mathrm{O} / \mathrm{THF}$, Ar, r.t., 3 h, ii. $\mathrm{HCl} 10 \%, 0{ }^{\circ} \mathrm{C}, 75 \%$; (b) i. TFA, DCM, Ar, r.t. 15 min, ii. $\mathrm{Et}_{3} \mathrm{SiH}, \mathrm{Ar}$, r.t., 1 h, iii. $\mathrm{H}_{2} \mathrm{O}, 0^{\circ} \mathrm{C}, \mathbf{2 4 : 7 2} \%, 27: 56 \%$; (c) i. $\mathrm{BBr}_{3}, \mathrm{DCM}^{\circ}$, r.t., ii. $\mathrm{H}_{2} \mathrm{O}, 0{ }^{\circ} \mathrm{C}, 97 \%$; (d) $n$-BuLi, THF, $-80{ }^{\circ} \mathrm{C}$; e) i. adamantanone, $-80{ }^{\circ} \mathrm{C}$ ii. $\mathrm{H}_{2} \mathrm{O}, 0{ }^{\circ} \mathrm{C}, 45 \%$; (f) $\mathrm{H}_{2} / 10 \% \mathrm{Pd}-\mathrm{C}$, EtOAc, $2 \mathrm{~h}$, almost quant. yield.
The first route involves the reaction of 4-methoxyphenylmagnesium bromide with 2-adamantanone (22) to give the carbinol 23, which was then reduced to the 2-(4-methoxyphenyl)adamantane (24). O-demethylation of 24 led to the desired phenol 19 in $52 \%$ overall yield (from adamantanone 22). The second reaction pathway involved the lithiation of the aryl bromide 25, [26] which was added to the 2-adamantanone (22) to give the 2-(4-(benzyloxy)phenyl)adamantan-2-ol (26). This was sequentially reduced and hydrogenated to give 4-(2-adamantyl)phenol (19) in $25 \%$ overall yield (from the aryl bromide 30 ).

The $N$-substituted-5-nitrofuran-2-carboxamides 3a-c were prepared as shown in Scheme 5.

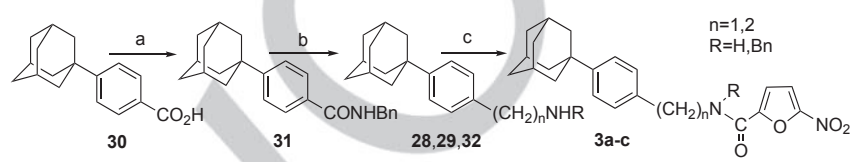

Scheme 5. Reagents and conditions: (a) i. $\mathrm{SOCl}_{2}$, reflux, $1 \mathrm{~h}$, ii. benzylamine, THF, r.t., 2 h, $83 \%$; (b) i. LiAlH ${ }_{4}$, THF, reflux, 2 h ii. EtOH, $\mathrm{H}_{2} \mathrm{O}, \mathrm{NaOH} 10 \%, 0{ }^{\circ} \mathrm{C}$ $83 \%$; (c) i. 5-nitro-2-furoic acid, $\mathrm{SOCl}_{2}$, reflux, 1 h, ii. appropriate amine $\mathbf{2 8}, \mathbf{2 9}$ 32 , acetone/pyridine, dropwise, r.t., $1 \mathrm{~d}, 43-53 \%$.

4-(1-Adamantyl)benzoic acid (30) [18] was converted to the 4-(adamant-1-yl)- $N$-benzylbenzamide (31), which was then reduced to the respective benzylamine $\mathbf{3 2}$. The methanamine $\mathbf{2 8}$, ${ }^{[27]}$ the ethanamine $29{ }^{[27]}$ and the benzylamine 32 were coupled with the intermediate, 5-nitro-2-furoic chloride, to afford the desired amides 3a-c, as shown in Scheme 5.

The new nifurtimox-adamantane adducts were tested for their activity against the bloodstream form Trypanosoma brucei and Trypanosoma cruzi epimastigotes and the results are shown in Tables 1 and 2.

Table 1. Anti-T. brucei screening of nifurtimox-adamantane derivatives.

\begin{tabular}{ccccc}
\hline Cmpd & $\begin{array}{c}\boldsymbol{T} \text {. brucei } \\
\mathrm{EC}_{50}(\mathbf{n M})^{[\mathrm{a}]}\end{array}$ & $\begin{array}{c}\boldsymbol{T} \text {. brucei } \\
\mathrm{EC}_{90}(\mathbf{n M})^{[\mathrm{a}]}\end{array}$ & $\begin{array}{c}\text { L6 cells } \\
\mathrm{EC}_{50}(\boldsymbol{\mu} \mathbf{M})^{[\mathrm{a}]}\end{array}$ & $\begin{array}{c}\text { S.I. } \\
\mathrm{EC}_{50} \mathbf{L 6 / \mathbf { T b } ^ { [ b ] }}\end{array}$ \\
\hline la & $19.0 \pm 1.00$ & $28.0 \pm 4.00$ & $1.85 \pm 0.08$ & 95 \\
Ib & $11.0 \pm 0.90$ & $23.9 \pm 0.60$ & $8.49 \pm 0.17$ & 770 \\
Ic & $15.9 \pm 1.80$ & $36.9 \pm 12.4$ & $8.28 \pm 0.24$ & 520 \\
Id & $29.0 \pm 1.00$ & $35.0 \pm 1.00$ & $3.04 \pm 0.21$ & 105 \\
2a & $41.0 \pm 3.00$ & $81.0 \pm 18.0$ & - & - \\
2b & $12.4 \pm 0.40$ & $25.2 \pm 1.90$ & $2.90 \pm 0.40$ & 235 \\
2c & $17.4 \pm 1.80$ & $41.2 \pm 5.90$ & $3.74 \pm 0.36$ & 215 \\
2d & $87.0 \pm 5.00$ & $172 \pm 9.00$ & - & - \\
a & $881 \pm 66.0$ & $1230 \pm 20.0$ & $14.5 \pm 0.40$ & 16 \\
3b & $353 \pm 38.0$ & $488 \pm 10.0$ & $4.06 \pm 0.23$ & 12 \\
3c & $322 \pm 17.0$ & $653 \pm 51.0$ & $1.38 \pm 0.15$ & 4.3 \\
Nifurtimox & $2510 \pm 90[28]$ & & &
\end{tabular}

[a] $\mathrm{EC}_{50}$ and $\mathrm{EC}_{90}$; concentration that inhibits growth by $50 \%$ and $90 \%$, respectively. [b] S.I.; selectivity index, the ratio of $\mathrm{EC}_{50}$ values obtained with $\mathrm{L} 6$ cells and T. brucei.

Table 2. Anti-T. cruzi screening of nifurtimox-adamantane derivatives.

\begin{tabular}{ccccc}
\hline Cmpd & $\begin{array}{c}\text { T. cruzi } \\
\mathrm{EC}_{50}(\mathbf{n M})^{[\mathrm{a}]}\end{array}$ & $\begin{array}{c}\text { T. cruzi } \\
\mathrm{EC}_{90}(\mathbf{n M})^{[\mathrm{aa}]}\end{array}$ & $\begin{array}{c}\text { L6 cells } \\
\mathrm{EC}_{50}(\boldsymbol{\mu M})^{[\mathrm{a}]}\end{array}$ & $\begin{array}{c}\text { S.I. } \\
\mathrm{EC}_{50} \text { L6/Tc }^{[\mathrm{b}]}\end{array}$ \\
\hline la & $178 \pm 13.0$ & $404 \pm 81.0$ & - & - \\
lb & $33.9 \pm 2.00$ & $63.0 \pm 5.00$ & $8.49 \pm 0.17$ & 250 \\
Ic & $70.7 \pm 13.8$ & $193 \pm 7.00$ & $8.28 \pm 0.24$ & 115 \\
Id & $85.0 \pm 9.00$ & $196 \pm 7.00$ & - & - \\
2a & $170 \pm 5.00$ & $330 \pm 5.00$ & - & - \\
2b & $55.4 \pm 1.20$ & $112 \pm 3.00$ & $2.90 \pm 0.40$ & 52 \\
2c & $95.2 \pm 19.0$ & $245 \pm 14.0$ & $3.74 \pm 0.36$ & 39 \\
2d & $330 \pm 4.00$ & $873 \pm 9.00$ & - & - \\
3a & $16200 \pm 500$ & $26600 \pm 200$ & $14.5 \pm 0.40$ & 0.9 \\
3b & $770 \pm 89.0$ & $1880 \pm 43.0$ & $4.06 \pm 0.23$ & 5.3 \\
3c & $1550 \pm 140$ & $5340 \pm 190$ & $1.38 \pm 0.15$ & 0.9 \\
Nifurtimox & $3100 \pm 500^{[29]}$ & & &
\end{tabular}

[a] $\mathrm{EC}_{50}$ and $\mathrm{EC}_{90}$; concentration that inhibits growth by $50 \%$ and $90 \%$, respectively. [b] S.I.; selectivity index, the ratio of $\mathrm{EC}_{50}$ values obtained with $\mathrm{L} 6$ cells and $T$. cruzi.

It is apparent that the hydrazone nifurtimox-adamantane adducts $\mathbf{1 a - d}$ and $\mathbf{2 a - d}$ are more potent trypanocidals than the parent drug 
(I) and $3-9$ fold more effective against $T$. brucei than $T$. cruzi. The most active adduct, with the best selectivity, was the phenylacetoxy hydrazone 1b $\left(E_{50}=11 \pm 0.9 \mathrm{nM}\right.$ and $\left.\mathrm{SI}_{\mathrm{Tb}}=770\right)$. The spacer between the phenyl ring and the carbonyl group seems to have a significant impact on activity and cytotoxicity. It seems that the present structural modification comprising of a phenyl ring insertion between the adamantane core and the hydrazone side chain has improved the pharmacological characteristics of the new molecules, in terms of activity and toxicity, compared to the adamantane carbohydrazones (IV), we previously reported. ${ }^{[22]}$ The direct attachment of the hydrazone linker to the phenyl ring decreased the potency, and a one or two methylene spacer was associated with enhanced activity. Adducts $\mathbf{1 b}, \mathbf{1 c}$ and $\mathbf{2 b}, \mathbf{2 c}$ exhibited higher trypanocidal activity than analogues $\mathbf{1 a}$ and $\mathbf{2 a}$, respectively. Conversely, the replacement of one methylene by an oxygen atom had a detrimental impact on activity. The position of substitution on the adamantane core $(\mathrm{C}-1, \mathrm{C}-2)$, influenced cytotoxicity and the $\mathrm{C}-1$ substituted hydrazones $1 \mathrm{~b}\left(\mathrm{Sl}_{\mathrm{Tb}}=770\right)$ and 1c $\left(\left.S\right|_{T b}=520\right)$ were found to be more selective than the corresponding $\mathrm{C}-2$ substituted adducts $\mathbf{2 b}\left(\mathrm{SI}_{\mathrm{Tb}}=235\right)$ and $2 \mathrm{c}$ $\left(\mathrm{SI}_{\mathrm{Tb}}=215\right)$. The same pattern was also observed in the $T$. cruzi results. The third series of derivatives, the 5-nitro-furonic carboxamides 3a-c, exhibited reduced activity in comparison to the hydrazones, which implies that these analogues may follow the same mechanistic pathway as nifurtimox. ${ }^{[30-32]}$ This observation is in agreement with our previous test results on adamantane carbohydrazones (IV). ${ }^{[22]}$

In conclusion, the new nifurtimox-adamantane hydrazone adducts show higher trypanocidal potency than both the parent drug (I) and the adamantane carbohydrazones (IV). The optimum potency arose from the combination of 4-(adamant-1-yl)phenyl substitution and a two methylene spacer, whilst their 4-(adamant-2-yl)phenyl substituted isomers were almost equipotent but less selective.

\section{Experimental Section}

General procedure for the preparation of hydrazones 1a-d and 2a-d. A solution of the respective hydrazide $(0.96 \mathrm{mmol})$ in ethanol $(5 \mathrm{~mL})$ was added to a solution of 5-nitro-2-furaldehyde $(200 \mathrm{mg}, 1.42 \mathrm{mmol}$ ) in ethanol $(5 \mathrm{~mL})$. The mixture was stirred under Argon in darkness for $12 \mathrm{~h}$. The resulting precipitate was filtered, washed with a small amount of ethanol and dried to give the corresponding product as a yellow solid, which was recrystallized from chloroform.

\section{General procedure for the preparation of carboxamides 3a-c.}

5-Nitro-2-furoic acid $(240 \mathrm{mg}, 1.52 \mathrm{mmol})$ was treated with $\mathrm{SOCl}_{2}(10 \mathrm{~mL})$ at $60-65{ }^{\circ} \mathrm{C}$ for $60 \mathrm{~min}$. Excess $\mathrm{SOCl}_{2}$ was removed under reduced pressure and subsequently by azeotropic distillation with benzene $(5 \mathrm{~mL})$. The residue obtained was dissolved in anhydrous acetone $(10-15 \mathrm{~mL})$ and a solution of the respective amine $(1.44 \mathrm{mmol})$ in anhydrous pyridine $(2 \mathrm{~mL})$ was added dropwise under stirring. The reaction mixture was stirred at room temperature for $1 \mathrm{~d}$, the solvent removed in vacuo and the residue was treated with water. The resulting mixture was extracted with $\mathrm{CH}_{2} \mathrm{Cl}_{2}$ and the organic extracts washed with water, $10 \% \mathrm{sol}$. $\mathrm{HCl}$ and water. The combined organic phases were dried over $\mathrm{Na}_{2} \mathrm{SO}_{4}$ and the solvent evaporated. The crude product was purified by gradient flash column chromatography, using as eluent a mixture of $\mathrm{CH}_{2} \mathrm{Cl}_{2} / \mathrm{MeOH}$, to give the corresponding carboxamide as an orange viscous semi-solid, which was recrystallized from chloroform.

\section{Conflicts of interest}

The authors have no conflicts to declare.

\section{Acknowledgements}

We thank Dr. Dimitra Benaki, Department of Pharmacy, Division of Pharmaceutical Chemistry, National and Kapodistrian University of Athens for NMR experiments.

Keywords: 4-(adamant-1-yl)phenyl substitution • 4-(adamant-2yl)phenyl substitution $\cdot$ hydrazone $\cdot$ nifurtimox $•$ trypanocidal activity

\section{References:}

[1] L. E. Echeverria, C. A. Morillo, Infect. Dis. Clin. North Am. 2019, 33 (1), 119134. DOI: $10.1016 / j . i d c .2018 .10 .015$

[2] E. Bottieau, J. Clerinx, Infect. Dis. Clin. North Am. 2019, 33 (1), 61-77. DOI: 10.1016/j.idc.2018.10.003.

3] S. P. S. Rao, M. P. Barrett, G. Dranoff, C. J. Faraday, C. R. Gimpelewicz, A. Hailu, C. L. Jones, J. M. Kelly, J. K. Lazdins-Helds, P. Mäser, et al., ACS Infect. Dis. 2019, 5 (2), 152-157. DOI: 10.1021/acsinfecdis.8b00298.

[4] A. F. Francisco, S. Jayawardhana, M. D. Lewis, M. C. Taylor, J. M. Kelly, Parasitology. 2017, 144 (14), 1871-1880. DOI: 10.1017/S0031182017001469.

5] M. Keenan, J. H. Chaplin, Prog. Med. Chem. 2015, 54, 185-230. DOI: 10.1016/bs.pmch.2014.12.001.

[6] "Fexinidazole (HAT) - DNDi," available at https://www.dndi.org/diseasesprojects/portfolio/fexinidazole/, accessed on 20 February 2018.

[7] I. Papanastasiou, A. Tsotinis, G. B. Foscolos, S. R. Prathalingam, J. M. Kelly, J. Heterocycl. Chem. 2008, 45 (5), 1401-1406. DOI: 10.1002/jhet.5570450524.

8] I. Papanastasiou, A. Tsotinis, N. Kolocouris, S. R. Prathalingam, J. M. Kelly, J. Med. Chem. 2008, 51 (5), 1496-1500. DOI: 10.1021/jm7014292.

[9] I. Papanastasiou, G. B. Foscolos, A. Tsotinis, J. Ol, J. Ov, S. R. Prathalingam, J. M. Kelly, Heterocycles. 2008, 75 (8), 2043-2061.

10] G. Foscolos, I. Papanastasiou, A. Tsotinis, Lett. Org. Chem. 2008, 5 (1), 5759. DOI: 10.2174/157017808783330117.

[11] I. Papanastasiou, K. C. Prousis, K. Georgikopoulou, T. Pavlidis, E. Scoulica N. Kolocouris, T. Calogeropoulou, Bioorg. Med. Chem. Lett. 2010, 20 (18) 5484-5487. DOI: 10.1016/j.bmcl.2010.07.078.

12] I. Papanastasiou, G. Foscolos B., A. Tsotinis, J. Kelly M., African Trypanosomiasis: Clinical Symptoms, Diagnosis and Treatment, Nova Science Publishers, NY 2016.

[13] S. Riganas, I. Papanastasiou, G. B. Foscolos, A. Tsotinis, K. Dimas, V. N. Kourafalos, A. Eleutheriades, V. I. Moutsos, H. Khan, P. Margarita, et al., Med. Chem. Shariqah United Arab Emir. 2012, 8 (4), 569-586.

14] S. Riganas, I. Papanastasiou, G. B. Foscolos, A. Tsotinis, J. J. Bourguignon, G. Serin, J. F. Mirjolet, K. Dimas, V. N. Kourafalos, A. Eleutheriades, et al. Bioorg. Med. Chem. 2012, 20 (10), 3323-3331. DOI: 10.1016/j.bmc.2012.03.038

[15] S. Riganas, I. Papanastasiou, G. B. Foscolos, A. Tsotinis, G. Serin, J.-F Mirjolet, K. Dimas, V. N. Kourafalos, A. Eleutheriades, V. I. Moutsos, et al., J. Med. Chem. 2012, 55 (22), 10241-10261. DOI: 10.1021/jm3013008.

[16] A. Koperniku, I. Papanastasiou, G. B. Foscolos, A. Tsotinis, M. C. Taylor, J. M. Kelly, MedChemComm. 2013, 4 (5), 856-859. DOI: 10.1039/C3MD00081H.

[17] I. Papanastasiou, S. Riganas, G. B. Foscolos, A. Tsotinis, S. A. Akhtar, M. A. Khan, K. M. Rahman, D. E Thurston, Lett Org Chem 2014, 12, 319-323.

[18] A. Koperniku, A.-S. Foscolos, I. Papanastasiou, G. B. Foscolos, A. Tsotinis, D. Schols, Lett. Org. Chem. 2016, 13, 171-176.

[19] A.-S. Foscolos, I. Papanastasiou, A. Tsotinis, N. Kolocouris, G. B. Foscolos A. Vocat, S. T. Cole, Med. Chem. 2017, 13 (7), 670-681. DOI: $10.2174 / 1573406413666170125112709$

[20] I. Papanastasiou, in Ration. Drug Des. (Eds: T. Mavromoustakos, T. F. Kellici), Vol. 1824, Springer New York, New York, NY 2018

21] M.-O. Georgiadis, V. Kourbeli, V. Ioannidou, E. Karakitsios, Papanastasiou, A. Tsotinis, D. Komiotis, A. Vocat, S. T. Cole, M. C. Taylor, et al., Bioorg. Med. Chem. Lett. 2019, 29 (11), 1278-1281. DOI: 10.1016/j.bmcl.2019.04.010

[22] A.-S. Foscolos, I. Papanastasiou, G. B. Foscolos, A. Tsotinis, T. F. Kellici, T. Mavromoustakos, M. C. Taylor, J. M. Kelly, MedChemComm. 2016, 7 (6), 1229-1236. DOI: 10.1039/C6MD00035E.

23] K. Lee, J.-I. Goo, H. Y. Jung, M. Kim, S. K. Boovanahalli, H. R. Park, M.O. Kim, D.-H. Kim, H. S. Lee, Y. Choi, Bioorg. Med. Chem. Lett. 2012, 22 (24) 7456-7460. DOI: 10.1016/j.bmcl.2012.10.046

24] Y. Arredondo, M. Moreno-Mañas, R. Pleixats, Synth. Commun. 1996, 26 (21), 3885-3895. DOI: 10.1080/00397919608003808.

25] N. Basarić, I. Žabčić, K. Mlinarić-Majerski, P. Wan, J. Org. Chem. 2010 75 (1), 102-116. DOI: 10.1021/jo902004n

26] W.-J. Hu, X.-L. Zhao, M.-L. Ma, F. Guo, X.-Q. Mi, B. Jiang, K. Wen, Eur. J. Org. Chem. 2012, 2012 (7), 1448-1454. DOI: 10.1002/ejoc.201101599.

$27]$ C. Chyi Tseng, I. Handa, A. N. Abdel-Sayed, L. Bauer, Tetrahedron. 1988 44 (7), 1893-1904. DOI: 10.1016/S0040-4020(01)90332-1.

[28] B. S. Hall, C. Bot, S. R. Wilkinson, J. Biol. Chem. 2011, 286 (15), 1308813095. DOI: 10.1074/jbc.M111.230847.

[29] M. C. O. Campos, L. L. Leon, M. C. Taylor, J. M. Kelly, Mol. Biochem Parasitol. 2015, 201 (1), 83. DOI: 10.1016/j.molbiopara.2015.05.006.

[30] S. R. Wilkinson, M. C. Taylor, D. Horn, J. M. Kelly, I. Cheeseman, Proc. Natl. Acad. Sci. 2008, 105 (13), 5022-5027. DOI: 10.1073/pnas.0711014105.

[31] S. R. Wilkinson, J. M. Kelly, Expert Rev. Mol. Med. 2009, 11. DOI: 10.1017/S1462399409001252.

[32] S. Patterson, S. Wyllie, Trends Parasitol. 2014, 30 (6), 289-298. DOI: 10.1016/j.pt.2014.04.003. 
WILEY-VCH

\section{Entry for the Table of Contents}

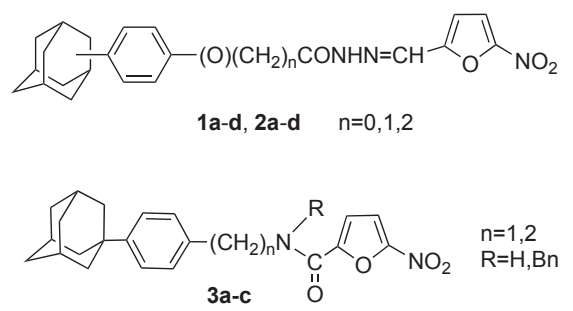

The insertion of a phenyl ring between the adamantane core and the hydrazone side chain of the new nifurtimox-adamantane adducts led to a higher trypanocidal activity and lower toxicity than the parent drug. The most active adduct with the best selectivity is the phenylacetoxy hydrazone $1 \mathrm{~b}\left(E C_{50}=11 \pm 0.9 \mathrm{nM}\right.$ and $\left.\mathrm{SI}_{\mathrm{Tb}}=770\right)$. 